The figures show that the thinnest specimens ("300" grades, $0 \cdot 020-0.025 \mathrm{~mm}$.) have, in single layers, relative retardations approaching, though in the main somewhat greater than that of the quarterwave mica plate, and a strip of the material bound between two glass slips by means of pieces of gummed label can in fact be used as a satisfactory substitute for this accessory. Three layers of the "300 Ordinary" mounted in the same way can be used in place of the unit retardation selenite plate, whilst a stepwise pile of strips affords an alternative to the mica stepped wedge or quartz wedge. The colours given by such a wedge appear to be quite normal.

It must be emphasised that the figures in the table refer to particular specimens. However, I have examined a number of other specimens taken from proprietary articles without finding wide deviations, and it seems that the birefringence of the material may be taken as roughly constant for a given thickness. It may happen that several specimens of the " 300 " thickness have to be tried before one is found from which a sensitive red can be built up. It may be mentioned that the higher birefringence of the moistureproof sample (last in table) as compared with others of the same thickness is not connected with its protective coating, for when this was dissolved off with a mixture of acetone and ethyl acetate, no apparent decrease in the birefringence was observed.

I am indebted to Messrs. Courtaulds for supplying graded samples and for information concerning the manufacture and properties of the material.

N. H. HAR'TSHORNE.

University College,
Swansea.

Jan. 10.

\section{Dimensions of Electric and Magnetic Units}

IF in a recent letter ${ }^{1}$ Sir James Henderson uses the word 'dimensions' in its customary sense of relation to the units of length, mass and time, he is arousing false hopes by suggesting the possibility of a 'discovery' of the dimensions of $\mu_{0}$ and $K_{0}$, for these dimensions, and not merely the numerical values of the quantities, are both completely arbitrary, depending upon the units of measurement which may be selected.

It has indeed been proved that $A^{2} / \mu_{0} K_{0}=c^{2}$ where $A$ is the quantity appearing in Ampère's equation and $c$ is the velocity of propagation of electromagnetic waves in a vacuum, but it still remains true that $\mu_{0}$ and $K_{0}$ may each be separately given any values whatever. In some systems of units which have been adopted or discussed, for example, in the Gaussian system, separate values have been assigned to each; in other systems, as in the "electrostatic' and in the 'electromagnetic' system, a value has been assigned to either $K_{0}$ or $\mu_{0}$ and the other obtained from it by an arbitrary assumption such as $A=1$, while other recently discussed systems are founded upon the adoption of two independent arbitrary assumptions from which the values of $K_{0}$ and $\mu_{0}$ can be deduced, but for purposes of measurement, as distinguished from historical or more or less sentimental considerations, a system depends only upon the values of $K_{0}$ and $\mu_{0}$ characteristic of it and not at all upon the methods by which these values were finally reached.

It is true that the electronic theory asserts that a magnet is not merely equivalent to but identical with a certain system of electrons in motion, but the argument that this identity necessarily requires the adoption of a system of units in which $\mu_{0}=1$ or in which $m L$ must be dimensionally equal to $i L^{2}$ is altogether unsound.

Sir James may readily convince himself of this by the examination of the following simple example. The modern form of the electronic theory obviously suggests the existence of a 'natural' system of electromagnetic measurement in which the unit of electric quantity will be the electron and the unit of magnetic moment will be the Bohr magneton. The 'natural' unit of current will be such that a current $i$ will involve the net passage of $i$ electrons per unit of time and the 'natural' unit of quantity of magnetism will be such that two equal and opposite quantities $m$ of magnetism separated by one unit of length will have a magnetic moment of $m$ magnetons. On this system it is obvious that $m L$ is dimensionally a numeric while the dimensions of $i L^{2}$ are $L^{2} / T$, and it is easily deducible that the dimensions of $K_{0}$ are $T^{2} / M L^{3}$ and of $\mu_{0}, T^{2} / M L^{5}$. The numerical values of these quantities will, of course, depend upon the units of length, mass and time adopted.

It is scarcely necessary to remark that the theoretical charms of such a 'natural' system are totally eclipsed by its practical inconveniences.

L. R. WILBERFORCE.

University of Liverpool.

Jan. 21.

${ }^{1}$ NATURE, 135, 105 ; 1935.

\section{Structure of the Caudal Fin of the Cod}

WITH reference to Dr. Whitehouse's suggestion ${ }^{1}$ that the caudal fin of the cod is of a normal homocercal type, may I state that I have just completed a study of the development of this fin, as a result of which I can affirm that, while it certainly preserves indications of its homocercal origin, it would be misleading to apply the term 'homocercal' to it in its present form.

Whereas in the homocercal fin practically the whole of the web is supported by morphologically ventral skeletal elements belonging to the hypochordal lobe, in the Gadoid fin the upper half of the web is supported by morphologically dorsal elements which arise in a dorsal fin fold, this growing back to fuse with a corresponding ventral fin fold and thus form the symmetrical web. Now Agassiz ${ }^{2}$ long ago showed that, because the homocercal fin developed as an exaggeration of heterocercy, the terminal 'axial lobe' of the embryonic caudal fin (in which is included the tip of the notochord) forms a small dorsal lobe to the developing definitive fin. $\mathrm{He}$ was, however, unable to find this lobe in the cod, as can be well understood from my own observations, which show that it is suppressed between the developing dorsal and ventral components of the fin; it is here, in other words, medial rather than dorsal in position.

For this and other reasons the term 'pseudocaudal' appears to me to be the most satisfactory designation for the Gadoid fin. A full report and discussion of my results will be published elsewhere.

Department of Zoology,

University College, Nottingham. Jan. 14.

1 NATURE, 185, 70, January 12, 1935.

2 Proc. Amer. Acad., 13 ; 1877.

E. J. W. Barrington. 